\title{
34. PHYSICAL PROPERTIES OF SEDIMENTS FROM THE MOUTH OF THE GULF OF CALIFORNIA, LEG 65, DEEP SEA DRILLING PROJECT ${ }^{1}$
}

\author{
Mario Gutiérrez-Estrada, ${ }^{2}$ Instituto de Ciencias del Mar y Limnología, Universidad Nacional \\ Autónoma de México, México 20, D.F., \\ and \\ Matthew H. Salisbury, Deep Sea Drilling Project, Scripps Institution of Oceanography, La Jolla, California
}

\begin{abstract}
The physical properties of 138 unconsolidated hemipelagic silty clays and turbidites from the mouth of the Gulf of California were analyzed in order to determine their grain size, shear strength, compressional wave velocity, porosity, specific gravity, wet-bulk density, water content, void ratio, and degree of saturation. As at other Deep Sea Drilling Project (DSDP) sites, the wet-bulk density, sonic velocity, and shear strength tended to increase with depth while the porosity and water content decreased in response to increasing compaction. The grain size and clay fraction varied irregularly with depth.

The wet-bulk density ranged from 1.34 to $2.58 \mathrm{~g} / \mathrm{cm}^{3}$, while the shear strength and compressional wave velocity ranged from 0.03 to 1.05 tons $/ \mathrm{ft} .{ }^{2}$ and 1.47 to $4.25 \mathrm{~km} / \mathrm{s}$, respectively. The porosity varied between 8 and $79 \%$, while the water content ranged from 28.0 to $175.6 \%$; most samples were effectively $100 \%$ saturated. The specific gravity ranged from 1.71 to 3.24 and showed a tendency to be directly related to the wet-bulk density and thus inversely related to porosity.

The physical properties of the hemipelagic sediments and turbidites are noticeably different, and the properties of both were modified by diagenesis near the basement.
\end{abstract}

\section{INTRODUCTION}

There are three principal methods of characterizing sediments: (1) microscopic and laboratory investigation of their constituents, (2) geochemical analysis, and (3) laboratory or field measurements of physical or engineering properties (Shepard, 1960; Richards, 1962). We shall here consider the physical properties (grain size, porosity, wet-bulk density, water content, specific gravity of solids, void ratio, degree of saturation, volume, shear strength, and compressional wave velocity) of 138 unconsolidated sediment samples of late Cenozoic age collected at four sites east of the tip of Baja California during DSDP Leg 65 (Fig. 1). The measurements reported were performed by the authors in the Laboratorio de Geología, Instituto de Ciencias del Mar y Limnología and Laboratorio de Sedimentología, Instituto de Geología, Universidad Nacional Autónoma de México. Terms and symbols used conform in general to those published in 1958 by the Joint Committee on Glossary of Terms and Definitions in Soil Mechanics of the American Society of Civil Engineers and the American Society for Testing Materials.

Measurements of wet-bulk density, porosity, compressional wave velocity, and shear strength were made at closely spaced intervals throughout the sediment column at all four sites. The values of wet-bulk density were determined at room temperature and pressure by means of the cylinder technique and are considered ac-

\footnotetext{
${ }^{1}$ Lewis, B. T. R., Robinson, P., et al., Init. Repts. DSDP, 65: Washington (U.S. Govt. Printing Office).

2 Present address: Estación de Investigaciones Mazatlán, Universidad Nationál Auton oma de Mexico, Mazatlán, Sinaloa, Mexico.
}

curate to $\pm 2 \%$ (Boyce, 1973). The porosities of the sediments were calculated from the densities of the samples before and after drying. The specific gravity of the solids, $G_{s}$, was determined from the relation,

$$
G_{s}=\frac{W_{s} G_{T}}{W_{s}-W_{1}+W_{2}}
$$

where $W_{s}$ is the weight of the sample after drying; $G_{T}$ is the specific gravity of distilled water at temperature $T$; $W_{1}$ is the weight of the sediment immersed in air-free distilled water; and $W_{2}$ is the weight of the volumetric flask and air-free water.

The water content, $W$, was computed from the relation,

$$
W=\frac{W_{w}}{W_{s}} \times 100
$$

and is the ratio of the weight of water in a given sample, $W_{w}$, to the weight of the sample after drying, $W_{s}$. As suggested by Richards (1962), we have also calculated the water content, $W C$, as a percentage of the total wet weight of the sample from the relation,

$$
W C=\frac{W_{w}}{W_{s}+W_{w}} \times 100
$$

and calculated the void ratio, $e$, which is the ratio of the volume of void space, $V_{v}$, to the volume of solid particles, $V_{s}$, in the sediment, as indicated in the relation, 


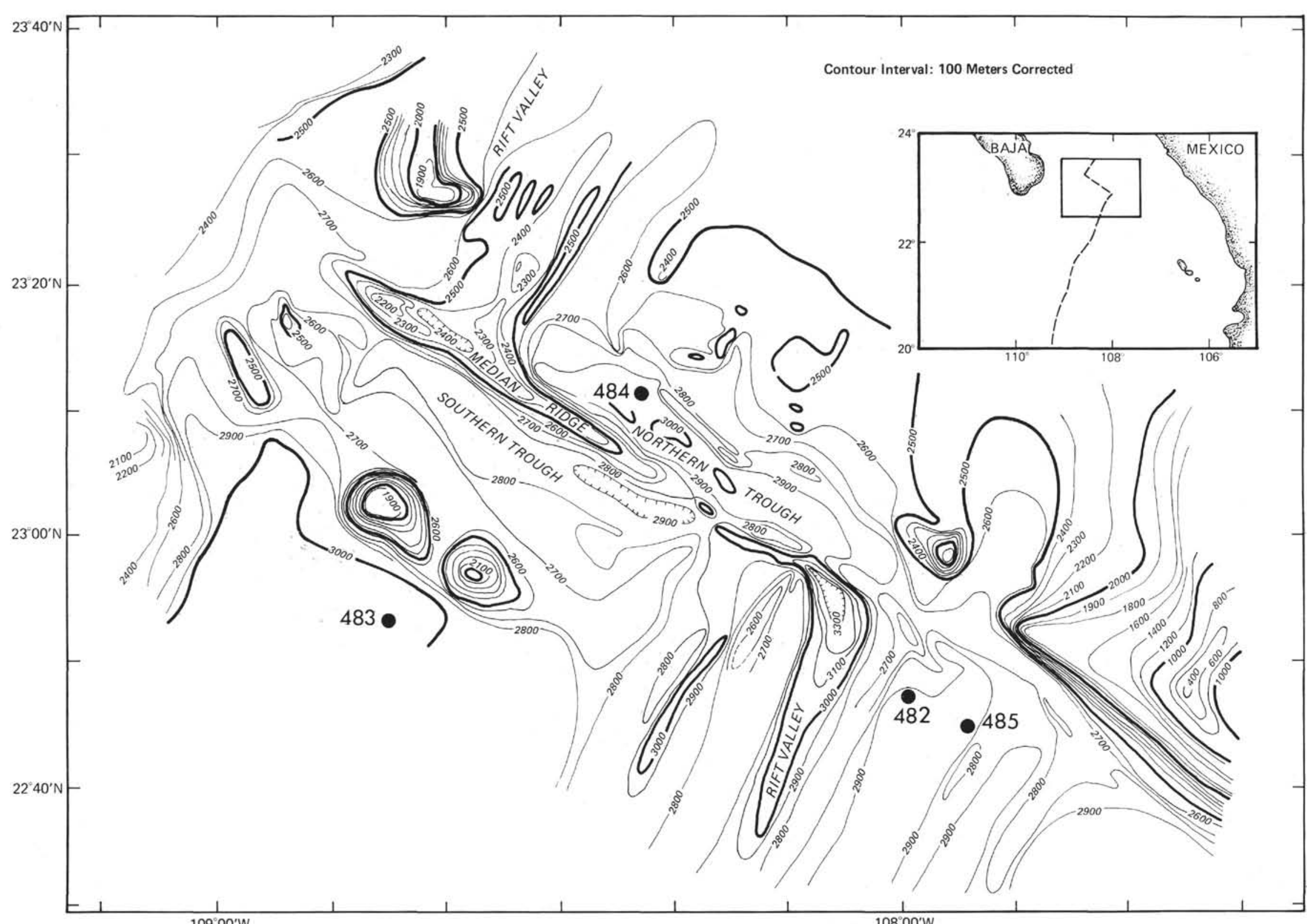




$$
e=\frac{V_{v}}{V_{s}} .
$$

This ratio, which is particularly applicable to watersaturated sediments, is determined in the laboratory from the relation,

$$
e=\frac{G_{s} \gamma_{w} V}{W_{s}}-1
$$

where $\gamma_{w}$ is the unit weight of water and $V$ is the volume of the sample.

The void ratio may also be recomputed at $100 \%$ saturation from the equation

$$
e_{\mathrm{sat}}=\frac{G_{s} W}{100}
$$

At $100 \%$ saturation, the water content is related to the porosity, $\phi$, and may be computed from the equation,

$$
W=\frac{\phi}{(100-\phi) G_{s}} \times 100 .
$$

The percent or degree of saturation, $S$, was computed from the relation

$$
S=\frac{W_{w}}{\gamma_{w}\left[V-\left(\frac{W_{S}}{G_{s} \gamma_{w}}\right)\right]} \times 100
$$

where the term in large brackets is the volume of voids, $V_{v}$.

The shear-strength measurements were made using a Torvane meter with interchangeable blade attachments. The compressional wave-velocity data were obtained at room temperature and pressure using the Hamilton Frame Velocimeter (Boyce, 1973). The grain-size information was determined by sieve and pipette techniques (Gutiérrez-Estrada, this volume). For samples with a cumulative percentage of less than $95 \%$ at $11 \phi$, the unsampled fine population was extrapolated to $100 \%$ at 14 $\phi$.

\section{RESULTS}

\section{Site $\mathbf{4 8 2}$}

The sediments at Site 482 (Fig. 2) consist predominantly of fine-grained hemipelagic silty clay and clayey silt containing $40 \%$ clay, with fine-grained turbidites containing up to $30 \%$ sand near the base of the section. As can be seen in Table 1, the values of wet-bulk density and porosity vary regularly with depth, the density increasing from approximately $1.6 \mathrm{~g} / \mathrm{cm}^{3}$ near the top of the column to about $1.9 \mathrm{~g} / \mathrm{cm}^{3}$ near the base. The only significant departures from this trend are observed in diagenetically altered sediments recovered near the basement where densities as high as $2.58 \mathrm{~g} / \mathrm{cm}^{3}$ and porosities as low as $13 \%$ are present.

With the exception of the altered sediments recovered in the lower parts of the section, which display compressional wave velocities as high as $4.25 \mathrm{~km} / \mathrm{s}$, the sediment velocities shown in Table 1 range for the most part between 1.5 and $1.6 \mathrm{~km} / \mathrm{s}$ and show a weak tendency to increase with depth. The velocities in the sediments between 40 and 70 meters sub-bottom could not be determined because of high signal attenuation caused by gas bubbles.

The shear strength was quite low throughout most of the column, ranging from about 0.2 to 0.4 tons $/ \mathrm{ft}^{2}{ }^{2}$ throughout most of the section except near the base, between 100 and 135 meters, where values as high as 0.8 tons $/ \mathrm{ft}^{2}{ }^{2}$ were encountered. Since the sediments are predominantly fine-grained throughout the column, the change near the base of the column is tentatively attributed either to a change in packing or to incipient diagenesis.

The specific gravities of the solids fall between 1.76 and 3.06 with most displaying values of 1.90 to 2.35 . The average for all of the samples is 2.15 . There is a tendency for the specific gravities to decrease with depth and thus to be inversely related to the wet-bulk density. For most of the sediments, the water content, $W$, ranges between 60.0 and $85.0 \%$, with the average being $62 \%$ and the extremes being $47 \%$ and $83 \%$. The degree of saturation fluctuates between $90 \%$ at a sub-bottom depth of 39.25 meters and $134 \%$ near the bottom of the sedimentary section.

\section{Site 483}

As can be seen in Figure 2, the sediments at Site 483 consist of soft to very soft, fossiliferous silty clay clayey silt and fine-grained turbidites. Between the mud line and a depth of 73 meters, the average grain size is $8.3 \phi$ and the clay fraction ranges from 30 to $70 \%$. Between 73 meters and the top of the basement at 109 meters, the average grain size is $8.3 \phi$ and the clay fraction averages $45 \%$. The sediments interlayered in the basement have an average grain size of 6.7 to $8.1 \phi$ and a clay fraction of 24 to $49 \%$.

With the exception of the shear strength, which increases from 0.04 to about 0.25 tons $/ \mathrm{ft}^{2}{ }^{2}$ in the upper 73 meters of the sediment column, the physical properties of the upper sediments show remarkably little variation with depth: the wet-bulk density ranges narrowly between 1.36 and $1.45 \mathrm{~g} / \mathrm{cm}^{3}$, the compressional wave velocity ranges from 1.47 to $1.49 \mathrm{~km} / \mathrm{s}$, the porosity ranges between 72 and $77 \%$, and the specific gravity of the solids fluctuates from 1.73 to 2.54 . Similarly, the water content, $W$, varies slightly from $124 \%$ to $172 \%$, the degree of saturation ranges from $108 \%$ to $120 \%$, and the void ratio ranges from 2.63 to 3.44 .

Between 73 meters and the basement, the physical properties of the sediments change markedly with depth in response to increasing compaction: the wet-bulk densities increase to about $1.7 \mathrm{~g} / \mathrm{cm}^{3}$, the compressional 


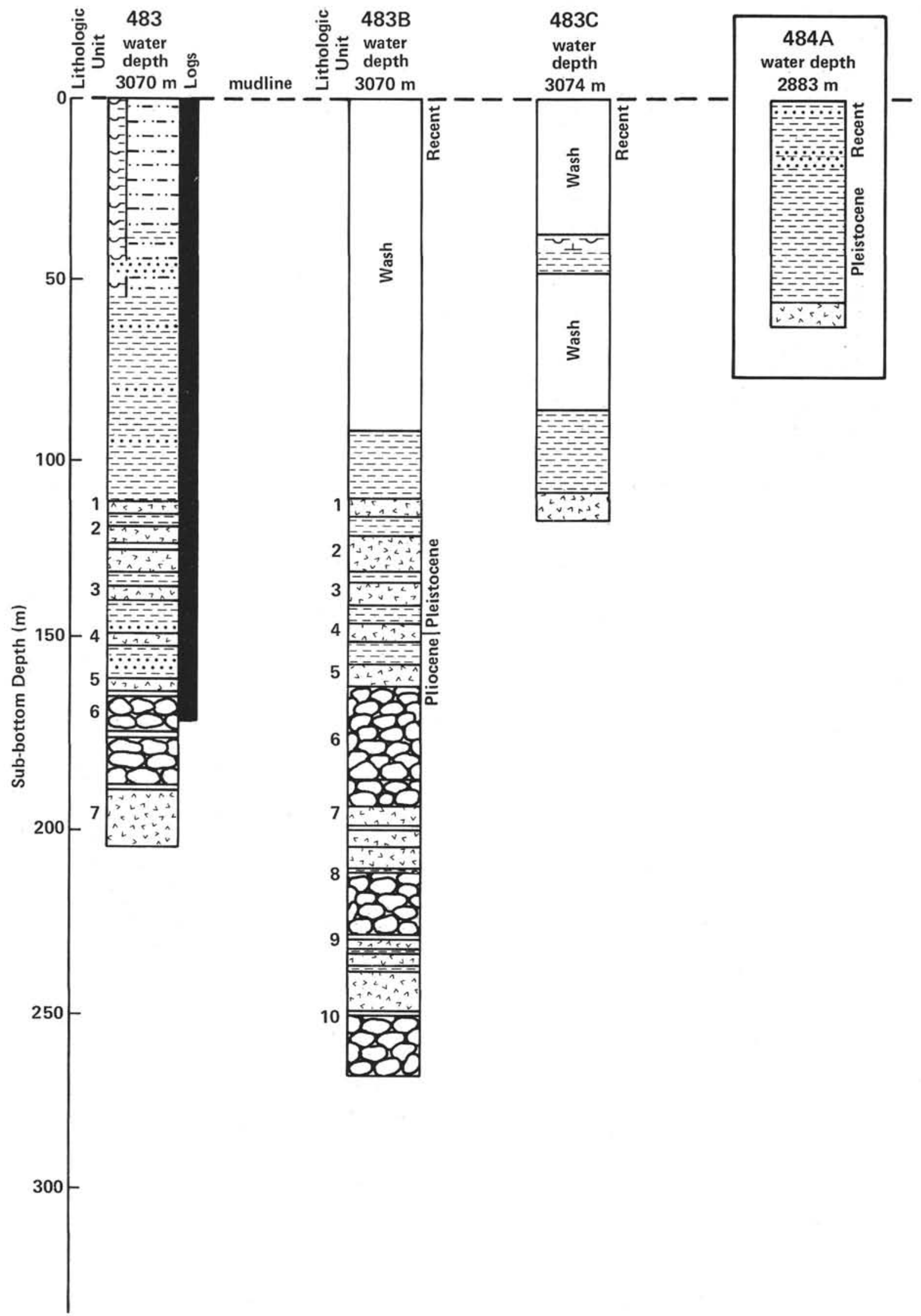

Figure 2. Lithologic columns for Sites 482, 483, 484, and 485. 


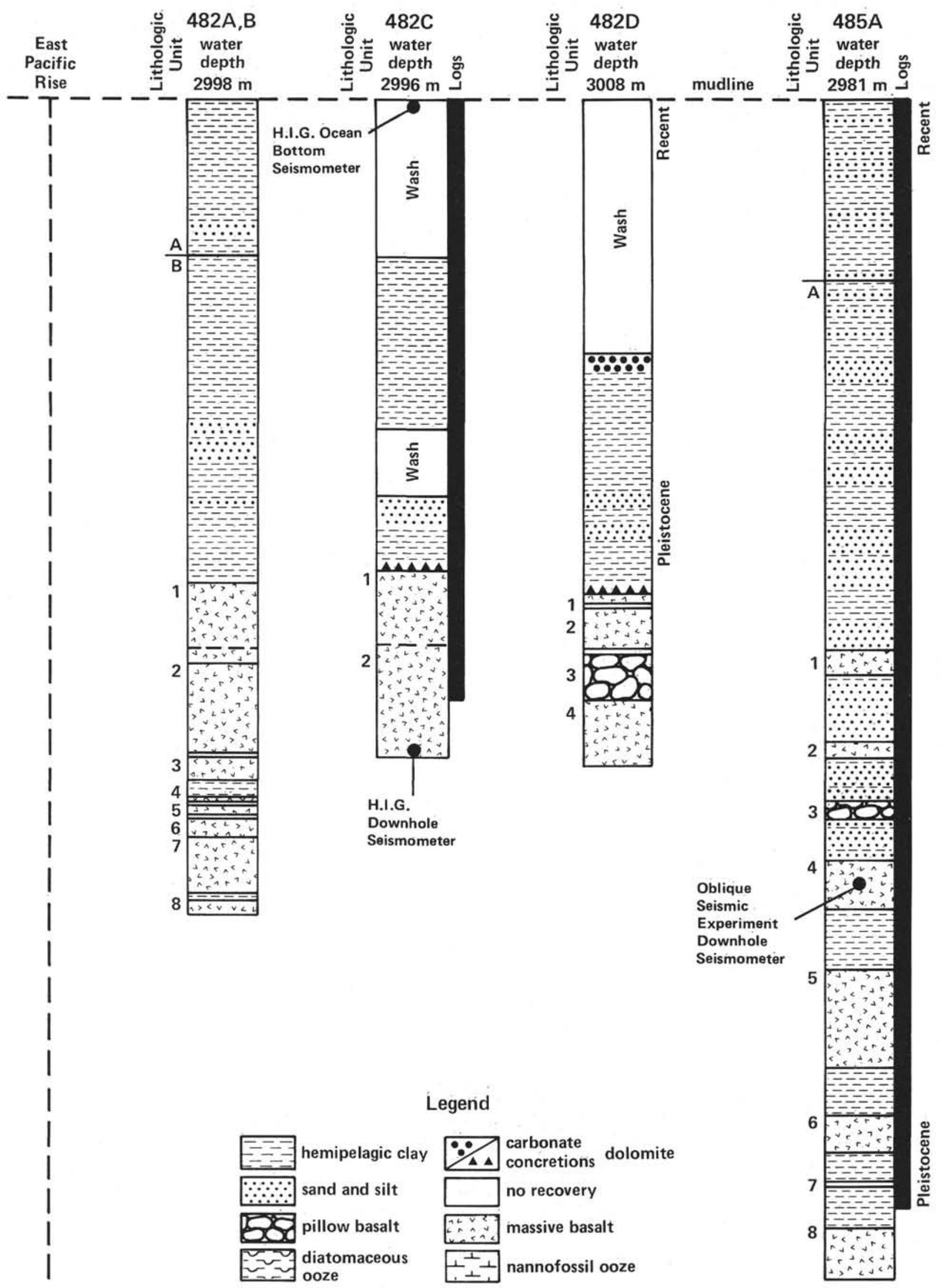

Figure 2. (Continued). 
Table 1. Physical properties of sediments, Leg 65 .

\begin{tabular}{|c|c|c|c|c|c|c|c|c|c|c|c|c|c|}
\hline \multirow{2}{*}{$\begin{array}{c}\text { Sample } \\
\text { (interval in cm) }\end{array}$} & \multirow{2}{*}{$\begin{array}{l}\text { Depth } \\
\text { (m) }\end{array}$} & \multirow{2}{*}{$\begin{array}{c}\text { Wet-Bulk } \\
\text { Density } \\
\left(\mathrm{g} / \mathrm{cm}^{3}\right)\end{array}$} & \multirow{2}{*}{$\begin{array}{l}\text { Porosity } \\
(\%)\end{array}$} & \multicolumn{2}{|c|}{$\begin{array}{l}\text { Water Content } \\
(\%)\end{array}$} & \multirow{2}{*}{$\begin{array}{l}\text { Specific } \\
\text { Gravity } \\
\text { of Solids }\end{array}$} & & $\begin{array}{l}\text { Degree of } \\
\text { Saturation }\end{array}$ & $\begin{array}{c}\text { Shear } \\
\text { Strength }\end{array}$ & $\begin{array}{l}P \text {-Wave } \\
\text { Velocity }\end{array}$ & $\begin{array}{l}\text { Clay } \\
\text { Fraction }\end{array}$ & $\begin{array}{c}\text { Mean } \\
\text { Grain Size }\end{array}$ & \\
\hline & & & & $W C$ & $\bar{W}$ & & Ratio & $(\%)$ & (tons $/ \mathrm{ft}^{2}{ }^{2}$ ) & $(\mathrm{km} / \mathrm{s})$ & & & Lithology \\
\hline $482 \mathrm{~A}$ & & & & & & & & & & & & & \\
\hline $\begin{array}{l}1-2,14-17 \\
1-4,80-90\end{array}$ & $\begin{array}{l}1.64 \\
5.30\end{array}$ & 1.57 & 64 & & & & & & & & 62 & 8.3 & Silty clay \\
\hline $2-2,18-21$ & 7.68 & & & & & & & & & & 39 & 7.4 & Clayey silt \\
\hline $2-4,60-68$ & 11.10 & 1.51 & 68 & & & & & & & & & & \\
\hline $4-4,37-49$ & 29.87 & 1.65 & 59 & & & & & & & & & & \\
\hline $\begin{array}{l}4-4,80-82 \\
4-4,25-27\end{array}$ & $\begin{array}{l}30.30 \\
39.25\end{array}$ & & & & & 3.06 & 1.43 & & & 1.52 & & 5.9 & Clayey silt, sandy silt \\
\hline $5-4,76-78$ & $\begin{array}{l}39.25 \\
39.76\end{array}$ & 1.66 & 59 & 32 & 47 & 3.06 & 1.43 & 90 & 0.23 & & 28 & & \\
\hline $482 \mathrm{~B}$ & & & & & & & & & & & & & \\
\hline $1-4,115-117$ & 49.65 & & & & & & & & 0.18 & & & & \\
\hline $1-4,139-141$ & 49.89 & 1.55 & 66 & 45 & 83 & 2.34 & 1.93 & 106 & & & 63 & 8.1 & Silty clay \\
\hline $2-4,35-41$ & $\begin{array}{l}58.35 \\
76.13\end{array}$ & 1.65 & 59 & 40 & 67 & 2.16 & 1.43 & 112 & 0.18 & & 40 & 7.5 & Clayey silt \\
\hline $\begin{array}{l}4-3,63-76 \\
4-3,95-97\end{array}$ & $\begin{array}{l}76.13 \\
76.45\end{array}$ & 1.63 & 61 & 42 & 72 & 2.17 & 1.56 & 112 & 0.19 & 1.54 & & & \\
\hline $5-3,5-7$ & 85.05 & & & & & & & & 0.26 & & 33 & 7.3 & Clayey silt \\
\hline $5-3,36-38$ & 85.36 & 1.62 & 61 & 41 & 71 & 2.21 & 1.56 & 110 & 0.20 & & 34 & 7.5 & Clayey silt \\
\hline $6-4,24-36$ & 96.24 & 1.75 & 53 & 32 & 48 & 2.35 & 1.12 & 107 & 0.08 & 1.58 & 12 & 4.9 & Silty sand \\
\hline $7-2,50-63$ & 103.00 & 1.70 & 56 & 38 & 61 & 2.09 & 1.27 & 115 & 0.57 & 1.49 & 46 & 8.4 & Clayey silt \\
\hline $8-4,71-74$ & 115.71 & 1.77 & 52 & 36 & 56 & 1.94 & 1.08 & 122 & & & 38 & 7.8 & Clayey silt \\
\hline $8-4,95-97$ & 115.95 & & & & & & & & 0.80 & & & & \\
\hline $8-4,116-118$ & 116.16 & & & & & & & & & 1.52 & & & \\
\hline $9-3,17-28$ & 123.17 & 1.75 & 53 & 36 & 57 & 1.99 & 1.13 & 119 & & & 29 & 7.7 & Clayey silt \\
\hline $10-5,75-77$ & 136.25 & & & & & & & & & 1.53 & & & Silty clay \\
\hline $\begin{array}{l}10-5,95-105 \\
19-1,31-33\end{array}$ & $\begin{array}{l}136.45 \\
193.31\end{array}$ & $\begin{array}{l}1.81 \\
1.9\end{array}$ & $\begin{array}{l}49 \\
44\end{array}$ & 33 & 50 & 1.91 & 0.96 & 124 & & 1.99 & 54 & 8.1 & Silty clay \\
\hline $482 \mathrm{C}$ & & & & & & & & & & & & & \\
\hline $8-1,72-75$ & 130.72 & 2.58 & 13 & & & & & & & 4.25 & & & Dolostone \\
\hline $9-1,8-10$ & 132.08 & & & & & & & & & 2.76 & & & Dolostone \\
\hline $482 \mathrm{D}$ & & & & & & & & & & & & & \\
\hline $1-3,35-37$ & 74.85 & 1.64 & & & & & & & 0.35 & & 42 & 7.5 & Clayey silt \\
\hline $1-3,120-131$ & 75.70 & 1.64 & 60 & & & & & & & 1.47 & & & \\
\hline $\begin{array}{l}2-3,95-100 \\
2-3,143-145\end{array}$ & 84.95 & 1.73 & 54 & 39 & 65 & 1.80 & 1.17 & 126 & & & 48 & 8.1 & Clayey silt \\
\hline $\begin{array}{l}2-3,143-145 \\
3-2,63-65\end{array}$ & 85.43 & & & & & & & & 0.35 & & & & \\
\hline $\begin{array}{l}3-2,63-65 \\
3-2,100-114\end{array}$ & $\begin{array}{l}92.63 \\
93.00\end{array}$ & & 56 & 42 & 72 & 1.76 & 1.27 & 128 & & 1.48 & 34 & 7.8 & Clayey silt \\
\hline $\begin{array}{l}3-2,100-114 \\
4-2,99-117\end{array}$ & 102.49 & 1.71 & & & & & & & & & 59 & 8.5 & $\begin{array}{l}\text { Clayey silt } \\
\text { Silty clay }\end{array}$ \\
\hline $\begin{array}{l}4-2,99-117 \\
5-1,49-54\end{array}$ & 109.99 & & & & & & & & 0.63 & 1.51 & & & \\
\hline $5-1,95-98$ & 110.45 & 1.72 & 55 & 38 & 63 & 1.95 & 1.22 & 120 & & & 32 & 7.4 & Clayey silt \\
\hline $6-2,13-16$ & 120.63 & 1.59 & & & & & & 134 & & & 23 & 6.6 & Clayey silt \\
\hline $6-2,40-43$ & 120.90 & 1.74 & 57 & & & & & & & 1.67 & & & \\
\hline $7-2,20-29$ & 130.20 & 1.80 & & & & & & & 0.40 & & 50 & 7.9 & Clayey silt \\
\hline $7-2,80-82$ & 130.80 & 1.69 & 59 & & & & & & & & & & \\
\hline $482 \mathrm{~F}$ & & & & & & & & & & & & & \\
\hline $4-3,37-39$ & 135.37 & 1.75 & 54 & & & & & & & 1.66 & & & \\
\hline 483 & & & & & & & & & & & & & \\
\hline $2-3,54-77$ & 4.54 & 1.39 & 76 & 60 & 153 & 2.07 & 3.15 & 111 & 0.04 & 1.47 & 55 & 8.2 & Silty clay \\
\hline $3-2,65-88$ & 12.65 & 1.44 & 73 & 58 & 135 & 1.99 & 2.69 & 113 & 0.05 & 1.48 & 68 & 9.3 & Silty clay \\
\hline $4-2,43-78$ & 21.93 & 1.41 & 74 & 57 & 135 & 2.11 & 2.84 & 109 & 0.11 & 1.49 & & 9.0 & Silty clay \\
\hline $6-2,75-97$ & 41.25 & 1.42 & 74 & 58 & 136 & 2.10 & 2.86 & 111 & 0.13 & 1.49 & & 8.3 & Clayey silt \\
\hline $7-4,88-103$ & 53.88 & 1.41 & 74 & 57 & 131 & 2.17 & 2.84 & 108 & 0.04 & 1.49 & & 8.4 & Silty clay \\
\hline $8-4,44-67$ & 62.94 & 1.42 & 74 & 58 & 136 & 2.09 & 2.84 & 111 & 0.11 & 1.49 & & 7.6 & Clayey silt \\
\hline $9-4,70-89$ & 72.70 & 1.43 & 73 & 55 & 124 & 2.18 & 2.70 & 108 & 0.25 & 1.49 & & 7.6 & Clayey silt \\
\hline $10-2,41-56$ & 78.91 & 1.54 & 66 & 49 & 97 & 1.99 & 1.94 & 115 & 0.18 & 1.49 & & 7.3 & Clayey silt \\
\hline $11-4,52-67$ & 91.52 & 1.57 & 64 & 46 & 84 & 2.10 & 1.77 & 112 & 0.20 & 1.51 & & 8.2 & Clayey silt \\
\hline $12-4,53-80$ & 101.03 & 1.70 & & & & & & & 0.48 & 1.51 & & 7.3 & Clayey silt \\
\hline $13-2,39-72$ & 107.39 & 1.64 & 60 & 45 & 82 & 1.83 & 1.49 & 123 & 0.69 & 1.50 & 37 & 7.6 & Clayey silt \\
\hline $18-4,24-57$ & 155.74 & 1.76 & & & & & & & 0.68 & 1.58 & & 8.1 & Clayey silt \\
\hline 483B & & & & & & & & & & & & & \\
\hline $1-3,102-111$ & 95.52 & 1.58 & 64 & 49 & 96 & 1.85 & 1.77 & 121 & & 1.52 & 46 & 8.2 & Clayey silt \\
\hline $1-3,128-143$ & 95.78 & 1.57 & 64 & 44 & 79 & 2.23 & 1.77 & 109 & 0.37 & & 33 & 7.5 & Clayey silt \\
\hline $1-5,80-82$ & 98.30 & 1.57 & 64 & 48 & 92 & 1.92 & 1.77 & 118 & & 1.52 & 57 & 8.6 & Silty clay \\
\hline $2-3,98-124$ & 104.98 & 1.65 & 60 & 44 & 78 & 1.92 & 1.49 & 121 & 0.46 & 1.57 & 43 & 8.1 & Clayey silt \\
\hline $2-4,60-62$ & 106.10 & 1.60 & 62 & 43 & 77 & 2.16 & 1.66 & 112 & & & 49 & 8.3 & Clayey silt \\
\hline $2-6,95-112$ & 109.45 & 1.70 & 56 & 40 & 67 & 1.90 & 1.27 & 122 & & 1.53 & 44 & 8.2 & Clayey silt \\
\hline $20-2,120-129$ & 211.20 & 1.93 & 45 & & & & & & & 1.93 & 24 & 6.7 & Clayey silt \\
\hline $25-2,5-24$ & 232.55 & 2.19 & 29 & & & & & & & 2.22 & 38 & 7.7 & Clayey silt \\
\hline $483 \mathrm{C}$ & & & & & & & & & & & & & \\
\hline $1-1,35-37$ & 38.85 & 1.45 & 72 & & & & & & & & 58 & 8.9 & Silty clay \\
\hline $1-2,96-98$ & 40.96 & 1.36 & 77 & 63 & 172 & 2.00 & 3.44 & 111 & & & 65 & 8.5 & Silty clay \\
\hline $1-3,55-57$ & 42.05 & 1.37 & 77 & 57 & 132 & 2.54 & 3.34 & 102 & & & 53 & 8.3 & Silty clay \\
\hline $1-4,60-62$ & 43.60 & 1.36 & 77 & 58 & 138 & 2.50 & 3.44 & 102 & & & 41 & 7.8 & Clayey silt \\
\hline $1-5,90-92$ & 45.40 & 1.44 & 72 & 60 & 152 & 1.73 & 2.63 & 120 & & & 46 & 8.1 & Clayey silt \\
\hline $2-3,9-12$ & 89.09 & 1.50 & 69 & 49 & 96 & 2.32 & 2.22 & 106 & & & 53 & 8.3 & Silty clay \\
\hline $2-7,90-107$ & 92.90 & 1.52 & & & & & & & & 1.48 & 56 & 8.6 & Silty clay \\
\hline
\end{tabular}


Table 1. (Continued).

\begin{tabular}{|c|c|c|c|c|c|c|c|c|c|c|c|c|c|}
\hline \multirow{2}{*}{$\begin{array}{c}\text { Sample } \\
\text { (interval in } \mathrm{cm} \text { ) }\end{array}$} & \multirow{2}{*}{$\begin{array}{l}\text { Depth } \\
\text { (m) }\end{array}$} & \multirow{2}{*}{$\begin{array}{l}\text { Wet-Bulk } \\
\text { Density } \\
\left(\mathrm{g} / \mathrm{cm}^{3}\right)\end{array}$} & \multirow{2}{*}{$\begin{array}{l}\text { Porosity } \\
(\%)\end{array}$} & \multicolumn{2}{|c|}{$\begin{array}{c}\text { Water Content } \\
\quad(\%) \\
\end{array}$} & $\begin{array}{l}\text { Specific } \\
\text { Gravity }\end{array}$ & Void & $\begin{array}{l}\text { Degree of } \\
\text { Saturation }\end{array}$ & $\begin{array}{c}\text { Shear } \\
\text { Strength }\end{array}$ & $\begin{array}{l}P \text {-Wave } \\
\text { Velocity }\end{array}$ & $\begin{array}{c}\text { Clay } \\
\text { Fraction }\end{array}$ & $\begin{array}{c}\text { Mean } \\
\text { Grain Size }\end{array}$ & \\
\hline & & & & $w C$ & $W$ & of Solids & Ratio & & & & & & Lithology \\
\hline $483 \mathrm{C}$ (cont.) & & & & & & & & & & & & & \\
\hline $2-7,15-17$ & 95.15 & 1.56 & 65 & 43 & 75 & 2.48 & 1.85 & 105 & & 1.55 & 31 & 7.2 & Clayey silt \\
\hline $3-2,75-77$ & 97.75 & 1.61 & 62 & 45 & 76 & 2.15 & 1.62 & 112 & & & 45 & 7.9 & Clayey silt \\
\hline $\begin{array}{l}3-2,105-107 \\
4-1,19-21\end{array}$ & 98.05 & & & & & & & & & 1.55 & 81 & 8.5 & \\
\hline $\begin{array}{l}4-1,19-21 \\
4-1,34-36\end{array}$ & $\begin{array}{l}105.19 \\
105.34\end{array}$ & 1.60 & 62 & 46 & 86 & 1.93 & 1.66 & 122 & & 1.67 & 51 & 8.5 & Silty clay \\
\hline $4-2,16-32$ & 106.66 & 1.47 & 71 & 54 & 115 & 2.13 & 2.45 & 113 & & 1.62 & 59 & 8.1 & Silty clay \\
\hline 484 & & & & & & & & & & & & & \\
\hline $1-1,112-115$ & 1.12 & & & & & & & & & 1.49 & & & \\
\hline $1-2,107-124$ & 2.57 & 1.45 & 72 & 52 & 110 & 2.34 & 2.57 & 106 & 0.09 & & 38 & 7.6 & Clayey silt \\
\hline $1-4,12-30$ & 4.62 & 1.46 & 71 & 49 & 98 & 2.50 & 2.45 & 103 & 0.13 & 1.50 & 48 & 8.1 & Silty clay \\
\hline $484 \mathrm{~A}$ & & & & & & & & & & & & & \\
\hline $1-3,93-110$ & 3.93 & 1.42 & 74 & 61 & 156 & 1.82 & 2.84 & 117 & & & 51 & 8.2 & Silty clay \\
\hline $1-4,61-100$ & $5: 11$ & 1.50 & 69 & 49 & 95 & 2.33 & 2.22 & 106 & & & 54 & 8.1 & Clayey silt \\
\hline $2-4,99-116$ & 13.49 & 1.56 & & & & & & & & & 55 & 8.1 & Silty clay \\
\hline $2-5,80-94$ & 14.80 & 1.40 & 75 & 56 & 125 & 2.40 & 3.00 & 104 & & & 53 & 8.5 & Silty clay \\
\hline $3-4,105-142$ & 23.05 & 1.50 & 69 & 53 & 113 & 1.97 & 2.22 & 121 & 0.25 & 1.68 & 67 & 9.1 & Silty clay \\
\hline $3-6,4-15$ & 25.04 & 1.50 & 69 & 54 & 118 & 1.88 & 2.22 & 118 & 0.24 & 1.47 & 58 & 8.5 & Silty clay \\
\hline $4-3,23-36$ & 30.23 & 1.47 & 71 & 58 & 136 & 1.80 & 2.44 & 120 & 0.18 & 1.48 & 49 & 8.2 & Silty clay \\
\hline $4-4,44-68$ & 31.94 & 1.44 & 72 & 59 & 143 & 1.83 & 2.62 & 117 & 0.11 & 1.48 & 48 & 8.5 & Clayey silt \\
\hline $5-4,65-87$ & 41.65 & 1.43 & 73 & 55 & 125 & 2.17 & 2.70 & 109 & 0.16 & 1.63 & & & \\
\hline $6-4,127-142$ & 51.77 & 1.50 & 69 & 53 & 115 & 1.94 & 2.22 & 117 & 0.39 & 1.48 & 49 & 8.5 & Clayey silt \\
\hline $6-5,13-32$ & 52.13 & 1.46 & 71 & 54 & 119 & 2.05 & 2.44 & 112 & 0.33 & 1.44 & 52 & 8.4 & Silty clay \\
\hline 485 & & & & & & & & & & & & & \\
\hline $1-1,109-113$ & 1.09 & & & & & & & & 0.03 & & & & \\
\hline $1-2,64-77$ & 2.14 & 1.34 & 79 & 64 & 176 & 2.15 & 3.77 & 108 & 0.05 & 1.50 & 30 & 6.8 & Clayey silt \\
\hline $\begin{array}{l}2-1,73-77 \\
2-2,102-105\end{array}$ & $\begin{array}{l}3.73 \\
5.52\end{array}$ & & & & & & & & 0.07 & & & & \\
\hline $\begin{array}{l}2-2,102-105 \\
2-3,71-110\end{array}$ & $\begin{array}{l}5.52 \\
6.71\end{array}$ & 1.46 & 71 & 12 & 132 & & & & 0.05 & 150 & 62 & 8.5 & Silty clay \\
\hline $3-2,119-121$ & 15.19 & & & & & 1.85 & 2.44 & 117 & $\begin{array}{l}0.18 \\
0.10\end{array}$ & 1.50 & 02 & 0.0 & Sing chay \\
\hline $3-4,22-24$ & 17.22 & & & & & & & & 0.14 & & & & \\
\hline $3-6,95-110$ & 20.95 & 1.49 & 69 & 53 & 111 & 2.01 & 2.22 & 114 & 0.14 & 1.50 & 38 & 7.9 & Clayey silt \\
\hline $4-2,110-112$ & 24.60 & & & & & & & & 0.22 & & & & \\
\hline $4-3,32-47$ & 25.32 & 1.50 & 69 & 49 & 98 & 2.27 & 2.22 & 108 & 0.25 & 1.51 & 48 & 8.3 & Clayey silt \\
\hline $5-2,44-46$ & 33.44 & & & & & & & & 0.19 & & & & \\
\hline $5-2,86-88$ & 33.86 & & & & & & & & 0.25 & & & & \\
\hline $5-4,36-47$ & 36.36 & 1.74 & & & & & & & 0.10 & 1.57 & & & \\
\hline $5-4,124-137$ & 37.24 & 1.59 & 63 & 44 & 79 & 2.14 & 1.70 & 112 & 0.30 & 1.53 & 43 & 8.1 & Clayey silt \\
\hline $6-2,82-84$ & 43.32 & & & & & & & & 0.20 & & & & \\
\hline $6-4,120-122$ & 46.70 & & & & & & & & 0.13 & & & & \\
\hline $6-5,138-140$ & 48.38 & & & & & & & & 0.17 & & & & \\
\hline $6-7,3-19$ & 50.03 & 1.54 & 66 & 45 & 82 & 2.37 & 1.94 & 105 & 0.33 & 1.48 & 43 & 8.0 & Clayey silt \\
\hline $485 \mathrm{~A}$ & & & & & & & & & & & & & \\
\hline $1-2,70-72$ & 52.70 & & & & & & & & 0.32 & & & & \\
\hline $1-4,63-65$ & 55.63 & & & & & & & & 0.29 & & & & \\
\hline $1-6,130-145$ & 59.30 & 1.61 & 62 & 44 & 80 & 2.05 & 1.62 & 115 & 0.46 & 1.52 & 42 & 8.2 & Clayey silt \\
\hline $2-3,107-109$ & 64.07 & & & & & & & & 0.35 & & & & \\
\hline $2-4,48-59$ & 64.98 & 1.65 & 59 & 46 & 84 & 1.71 & 1.43 & 128 & 0.48 & 1.49 & 37 & 7.3 & Clayey silt \\
\hline $3-2,110-118$ & 72.10 & 1.57 & 64 & 47 & 89 & 1.99 & 1.77 & 116 & 0.45 & & 36 & 7.4 & Clayey silt \\
\hline $3-3,41-44$ & 72.91 & & & & & & & & & 1.45 & & & \\
\hline $4-1,42-70$ & 79.42 & 1.65 & 59 & 42 & 73 & 1.97 & 1.43 & 118 & 0.54 & 1.49 & 40 & 7.9 & Clayey silt \\
\hline $5-3,110-123$ & 92.60 & 1.82 & & & & & & & 0.53 & 1.55 & 40 & 7.9 & Clayey silt \\
\hline $5-4,32-48$ & 93.32 & 1.83 & 48 & 29 & 41 & 2.24 & 0.92 & 111 & 0.38 & 1.57 & 26 & 6.6 & Clayey silt \\
\hline $6-3,42-77$ & 101.42 & 1.83 & 48 & 31 & 46 & 2.03 & 0.92 & 119 & 0.55 & 1.61 & 35 & 7.2 & Clayey silt \\
\hline $6-3,100-107$ & 102.00 & 1.80 & 50 & 24 & 31 & 3.24 & 1.00 & 85 & & 1.55 & 8 & 4.8 & Sandy silt \\
\hline $7-2,7-25$ & 109.07 & 1.73 & 54 & 38 & 61 & 1.91 & 1.17 & 122 & 0.69 & 1.69 & 44 & 8.2 & Clayey silt \\
\hline $8-2,103-125$ & 119.53 & 1.80 & 50 & 34 & 52 & 1.92 & 0.99 & 123 & 1.05 & 1.60 & 48 & 8.4 & Clayey silt \\
\hline $9-2,87-104$ & 128.87 & 1.93 & 42 & & & & & & 0.06 & 1.73 & 15 & 5.7 & Sandy silt \\
\hline $10-1,121-130$ & 137.21 & 1.64 & 60 & & & & & & 0.33 & & & & \\
\hline $10-2,62-78$ & 138.12 & 1.78 & 51 & 35 & 54 & 1.92 & 1.04 & 123 & 1.03 & 1.65 & 45 & 7.7 & Clayey silt \\
\hline $11-1,104-140$ & 146.54 & 1.78 & 53 & & & & & & & 1.67 & & & \\
\hline $18-1,103-112$ & 185.16 & 1.92 & & & & & & & 0.24 & & 10 & 5.3 & Silt \\
\hline $19-2,16-42$ & 189.66 & 1.85 & 47 & 29 & 41 & 2.17 & 0.88 & 114 & 0.40 & 1.54 & 8 & 4.8 & Sandy silt \\
\hline $19-2,78-137$ & 190.28 & 1.82 & 49 & 33 & 48 & 1.98 & 0.96 & 121 & & & 37 & 7.7 & Clayey silt \\
\hline $20-1,95-121$ & 193.45 & 1.83 & 51 & & & & & & & & & & \\
\hline $20, \mathrm{CC}-(21-33)$ & 194.56 & 2.57 & 8 & & & & & & & & & & Sandstone \\
\hline $22-1,77-80$ & 202.27 & 1.80 & 54 & & & & & & & & & & \\
\hline $22-3,138-143$ & 205.88 & 1.84 & 49 & & & & & & & & & & \\
\hline $26-1,51-118$ & 226.51 & 1.79 & 51 & 27 & 37 & 2.81 & 1.04 & 95 & & & 34 & 7.9 & Clayey silt \\
\hline $27-1,121-128$ & 231.71 & 1.81 & 50 & 27 & 36 & 2.65 & 0.96 & 101 & & & 44 & 8.2 & Clayey silt \\
\hline $28-1,113-134$ & 236.13 & 1.84 & 48 & 27 & 37 & 2.41 & 0.88 & 105 & & & 46 & 8.2 & Clayey silt \\
\hline $34-1,41-43$ & 277.41 & 1.93 & 42 & 22 & 28 & 2.58 & 0.72 & 101 & & & 19 & 6.1 & Silt \\
\hline $36-1,20-23$ & 295.20 & & & & & & & & & 2.35 & & & \\
\hline $36-1,101-140$ & 296.01 & 1.93 & 46 & & & & & & & & 49 & 81 & Tyyey silt \\
\hline $\begin{array}{l}37-1,41-70 \\
38-1,18-19\end{array}$ & $\begin{array}{l}304.41 \\
313.18\end{array}$ & 1.87 & 46 & 23 & 29 & 2.91 & 0.85 & 92 & & 2.17 & 49 & 8.1 & Clayey silt \\
\hline
\end{tabular}


wave velocities increase to values as high as $1.67 \mathrm{~km} / \mathrm{s}$, and the shear strength rises to about 0.7 tons $/ \mathrm{ft} .{ }^{2} \mathrm{Simi}-$ larly, the porosity decreases to about $55 \%$, the water content decreases rapidly to values as low as $67 \%$, the degree of saturation increases to about $120 \%$, and the void ratio decreases to 1.27 . At the same time, the specific gravity of the solids varies irregularly between 1.73 and 2.54 .

The physical properties of the sediments interlayered in the basement are quite variable but, in general, reflect increased compaction and incipient diagenesis. The wetbulk density, for example, ranges between 1.76 and 2.19 $\mathrm{g} / \mathrm{cm}^{3}$, the compressional wave velocity varies between 1.58 and $2.22 \mathrm{~km} / \mathrm{s}$, and the porosity ranges between 29 and $45 \%$.

\section{Site $\mathbf{4 8 4}$}

The sediments at Site 484 consist of 55 meters of hemipelagic clayey silt and silty clay with interlayered beds of sandy silt. The average clay fraction is $52 \%$ and the mean grain size is $8.3 \phi$.

The physical properties of the sediments recovered in Holes 484 and $484 \mathrm{~A}$ are somewhat anomalous, both with respect to those observed at Sites 482 and 483 and to those observed at DSDP sites in general. Although the average wet-bulk density, $1.45 \mathrm{~g} / \mathrm{cm}^{3}$, is fairly typi$\mathrm{cal}$ of sediments recovered near the mud line, the maximum compressional wave velocity observed at Site 484 , $1.68 \mathrm{~km} / \mathrm{g}$, and the average shear strength, 0.20 tons/ $\mathrm{ft}^{2}$, are unexpectedly high. Similarly, the average porosity, $72 \%$, the average water content, $111 \%$, and the average void ratio, 2.31 , are somewhat lower than expected. The average specific gravity of the solids and the average degree of saturation are 1.92 and $104 \%$, respectively. Although most of these values are not unusual, they are atypical of shallow sediments.

\section{Site $\mathbf{4 8 5}$}

The sediments overlying the basement at Site 485 consist of very poorly sorted hemipelagic clayey silt and silty clay with minor amounts of terrigenous silt and sandy silt. The average particle size ranges from $4.8 \phi$ to 8.5 , and the clay fraction ranges from 7 to $61 \%$, about an average of $35 \%$.

As at the other sites drilled on Leg 65, most of the physical properties change markedly with depth in response to compaction. The wet-bulk density, for example, increases irregularly from about $1.40 \mathrm{~g} / \mathrm{cm}^{3}$ at the mud line to about $1.80 \mathrm{~g} / \mathrm{cm}^{3}$ near the basement contact while the compressional wave velocity increases from 1.5 to about $1.7 \mathrm{~km} / \mathrm{s}$ and the shear strength increases from 0.03 tons $/ \mathrm{ft} .^{2}$ to values as high as 1.05 tons $/ \mathrm{ft} .^{2}$ Over this same interval, the porosity decreases from 79 to $53 \%$, the water content decreases from 176 to $54 \%$ and the void ratio diminishes from about 3.8 to 1.0. The specific gravity of the solids and the degree of saturation range irregularly with depth from 1.71 to 3.24 and from $85 \%$ to $128 \%$, respectively.

Within the relatively fine-grained $(\phi$ average $=7.0$ ) silty clay, clayey silt, and claystone found interlayered below the uppermost basalts (Fig. 2), the physical prop- erties continue to change with depth in response to increasing compaction, but much more slowly. The wetbulk density, velocity, and shear strength commonly reach values as high as $1.93 \mathrm{~g} / \mathrm{cm}^{3}, 2.17 \mathrm{~km} / \mathrm{s}$, and 0.4 tons $/ \mathrm{ft}^{2}{ }^{2}$, respectively, with more extreme values being observed in diagenetically altered samples. Similarly, the porosity decreases to about $46 \%$ and the water content decreases from about 41 to $29 \%$. The specific gravity of the solids, the degree of saturation, and the void ratio, however, vary irregularly with depth in this interval from 1.98 to $2.91,92$ to $121 \%$, and 0.72 to 1.04 , respectively.

\section{DISCUSSION}

Most of the sediments analyzed from Sites 482,483 , 484 , and 485 were hemipelagic clays with an average grain size of 6.5 to $9.2 \phi$ and minor turbidites composed of terrigenous material with an average grain size of 4.9 to $6.0 \phi$.

Practically all of the samples analyzed were sufficiently fine grained to make the 84th percentile.

As can be seen in Figures 3 and 4, both the void ratio and the porosity increase sharply with decreasing grain size. Thus the fine-grained sediments $(\phi>7.0)$ have high porosities, as indicated by the empirical boundary line shown in Figure 4. This result is similar to that obtained by Richards (1962).

The specific gravities of the solids analyzed range between 1.7 and 3.2 , with the majority of the values falling between 1.8 and 2.2 (Fig. 5). As can be seen in Figure 6 , the values of wet-bulk density range from a maximum of $2.6 \mathrm{~g} / \mathrm{cm}^{3}$ at $8.0 \%$ porosity to a minimum of

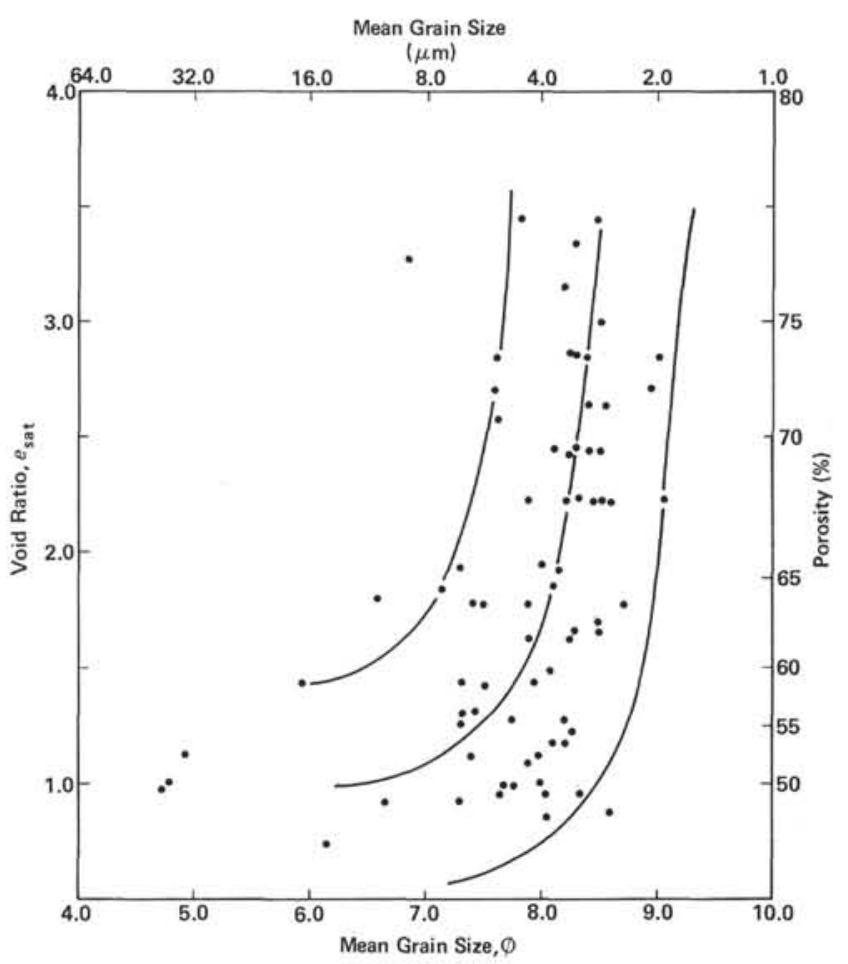

Figure 3. Void ratio vs. mean grain size for Leg 65 sediments. (Lines represent average and extreme values.) 


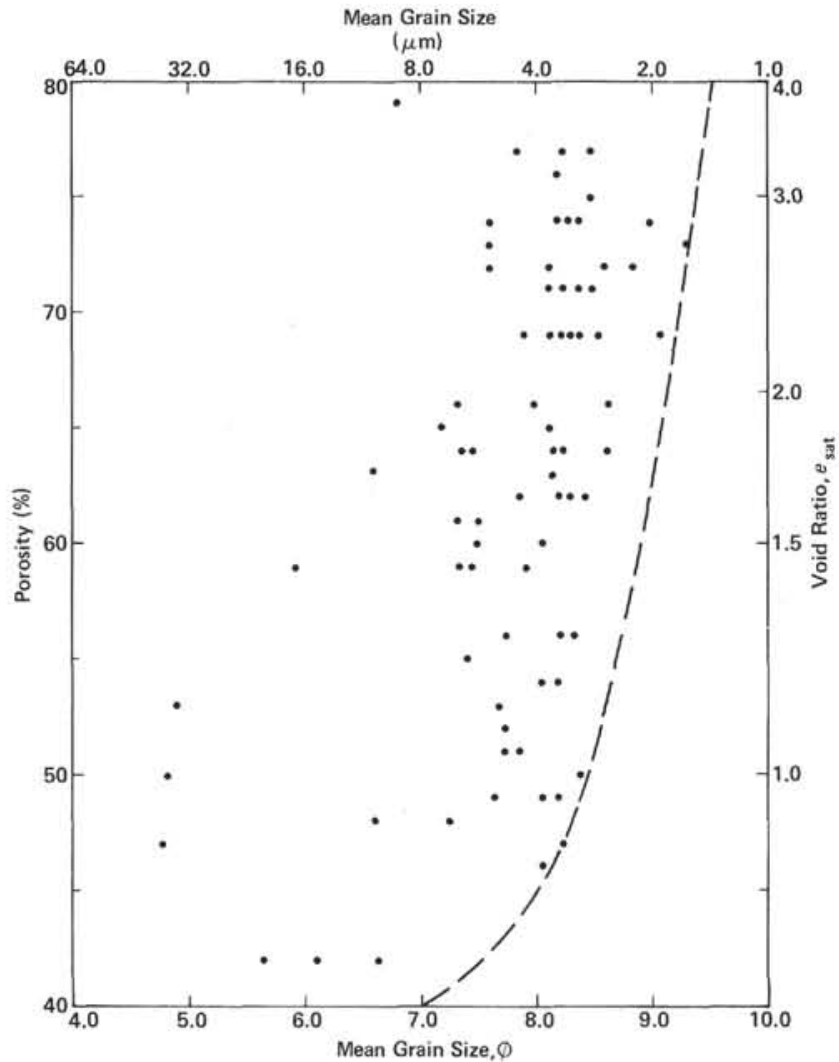

Figure 4. Porosity vs. mean grain size for Leg 65 sediments. (Dashed line represents minimum porosity curve.)

$1.34 \mathrm{~g} / \mathrm{cm}^{3}$ at $79.0 \%$ porosity and display the straightline relationship between wet-bulk density and porosity shown by Hamilton and Menard (1956), Nafe and Drake (1957) and Richards (1962). There is a slight tendency, however, for the specific gravity to increase with increasing wet-bulk density.

In general, the water content is inversely related to the depth of burial (Fig. 7). The lowest water content measured was $28 \%$ in a silt recovered from a sub-bottom depth of 277 meters in Hole $485 \mathrm{~A}$ and the highest was $176 \%$ in a clayey silt cored at about 2 meters depth in Hole 485. The scatter in the data is attributed to turbidites containing sand, silt, and reworked foraminifers which display anomalously high water contents. These layers also display lower shear strengths (about 0.35 tons $/ \mathrm{ft}^{2}{ }^{2}$ ) than the silty clays comprising most of the section and, when more cemented, display higher densities and velocities and lower porosities.

Practically all of the physical properties of unconsolidated sediments are related to the amount of interstitial water present in the sediments (Richards, 1962). All but three of the samples investigated, regardless of type and origin, were more than $95 \%$ saturated. Since the sediments are presumably $100 \%$ saturated, but the average measured degree of saturation is $111 \%$, the average measurement error is $11 \%$.

The wet-bulk densities and porosities range from 1.34 to $2.58 \mathrm{~g} / \mathrm{cm}^{3}$ and from 8 to $79 \%$, respectively. As at most DSDP sites, the wet-bulk density tends to increase,

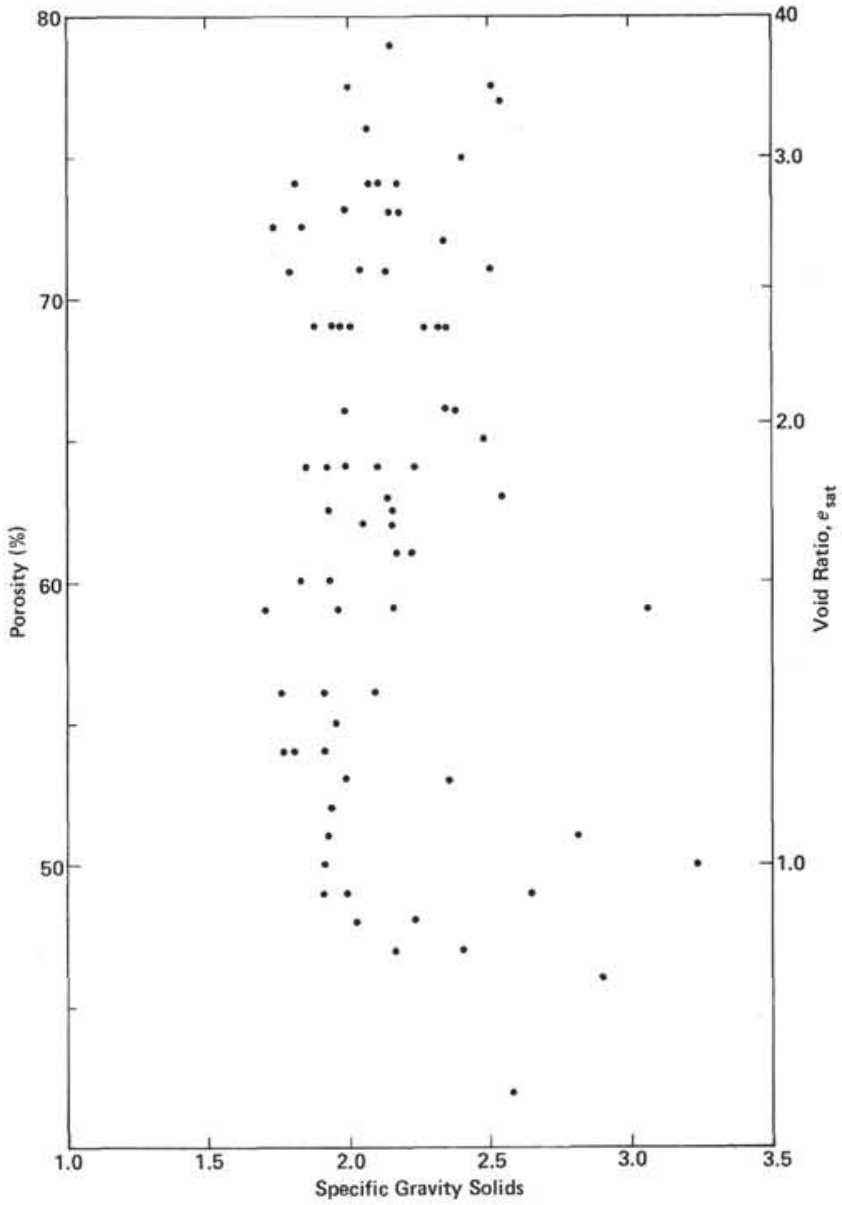

Figure 5. Porosity vs. specific gravity of solids for Leg 65 sediments.

and the porosity to decrease, with depth in the young sediments at the top of the section. The compressional wave velocities, which range between 1.5 and $1.6 \mathrm{~km} / \mathrm{s}$, are typical of weakly consolidated sediments, but higher values were found in the more compact sediments near the base of the sections. The sediments interlayered within the basement tend to have even higher densities and velocities and lower porosities than the sediments above basement in response to increased compaction and diagenesis.

\section{ACKNOWLEDGMENTS}

The writers wish to express their appreciation to the following persons from the Universidad Nacional Autónoma de México for their assistance in this study: A. Ayala-Castañares, Director del Instituto de Ciencias del Mar y Limnología; A. Laguarda-Figueras, ex-Director del Instituto de Ciencias del Mar y Limnología; D. Córdoba-Mendez, ex-Director del Instituto de Geologia; A. Castro; A. Pérez-Rojas and A. Galaviz-Solis, Instituto de Ciencias del Mar y Limnología, for their help in the laboratory; and E. León, for typing the manuscript.

\section{REFERENCES}

Boyce, R. E., 1973. Appendix I: Physical property methods. In Edgar, N. T., Saunders, J. B., et al., Init. Repts. DSDP, 15: Washington (U.S. Govt. Printing Office), 1115-1128.

Committee on Glossary of Terms and Definitions, 1958. Glossary of terms and definitions in soil mechanics, $J$. Soil Mechanics Foundations Div. (American Society of Civil Engineers), 84(SM 4):1-43. 
Hamilton, E. L., and Menard, H. W., 1956. Density and porosity of sea-floor surface sediments off San Diego, California. Am. Assoc. Pet. Geol. Bull., 40:754-761.

Nafe, J. E., and Drake, C. L., 1957. Variation with depth in shallow and deep water marine sediments of porosity, density and velocities of compressional and shear waves. Geophysics, 22:523-552.

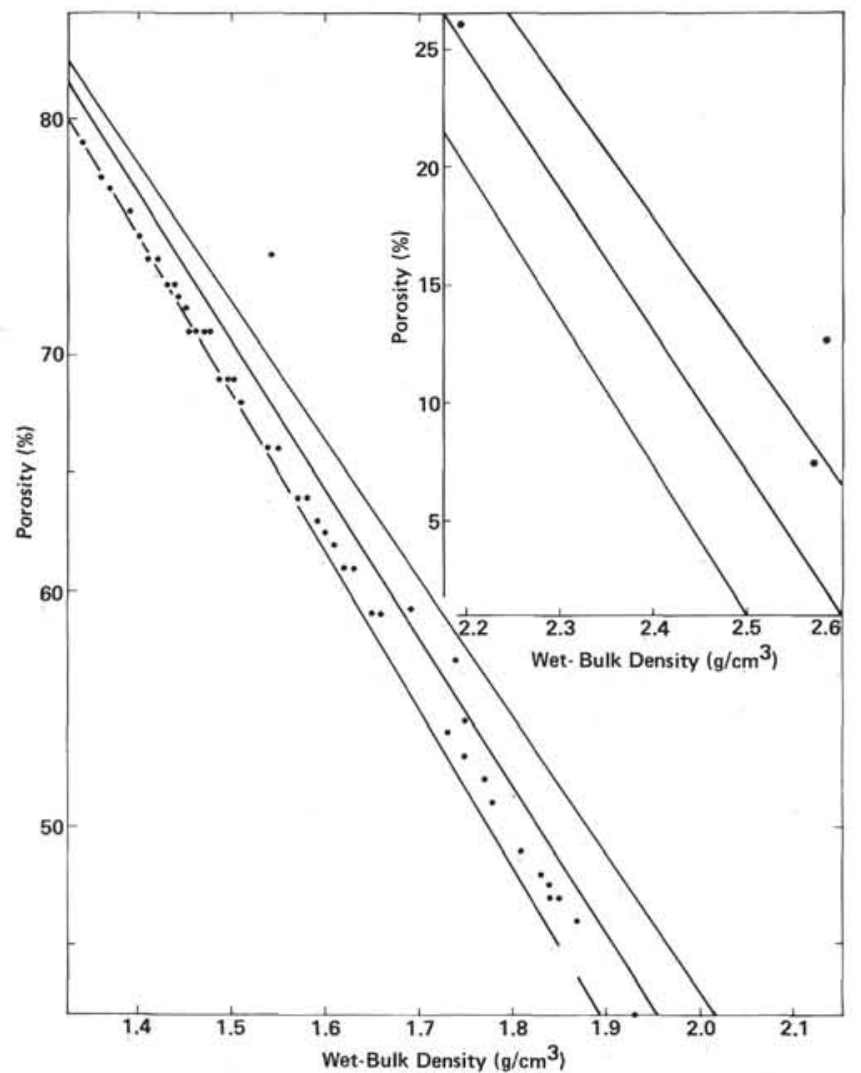

Figure 6. Porosity vs. wet-bulk density for Leg 65 sediments. (Lines of equal specific gravity shown for comparison.)
Richards, A. F., 1962. Investigations of Deep-Sea Sediment Cores, 11: Mass Physical Properties: Washington (U.S. Navy Hydrographic Office).

Shepard, F. P., 1960. Mississippi Delta: Marginal Environments, Sediments, and Growth. In Shepard, F. D., Phleger, F. B., and van Andel, Tj. H. (Eds.), Recent Sediments, Northwest Gulf of Mexico: Tulsa (Amer. Assoc. Petrol. Geol.), pp. 56-81.

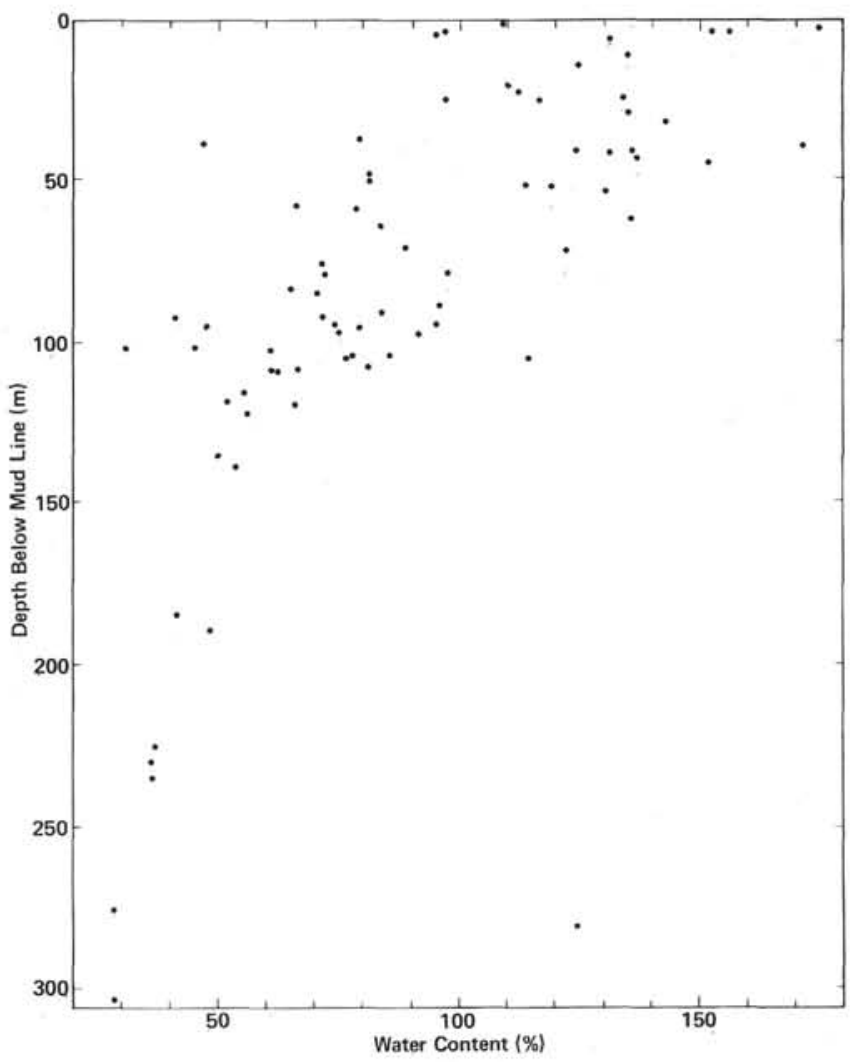

Figure 7. Water content vs. depth below mud line for Leg 65 sediments. 